\title{
Mechano-chemostats to study the effects of compressive stress on yeast
}

\author{
L. Holt ${ }^{1}$, O. Hallatschek ${ }^{2}$ and M. Delarue ${ }^{3}$
}

1. Institute for Systems Genetics, New York University Langone Medical Center, New York, NY 10016, USA

2. Department of Physics and Integrative Biology, University of California, Berkeley, CA 94720, USA

3. MILE, Laboratory for Analysis and Architecture of Systems, CNRS, 31400, Toulouse, France

\section{Introduction}

Cells habitually proliferate in a confined environment. For example, microbes often inhabit micrometer-sized pores (Warscheid \& Braams, 2000), and mammalian cells such as solid tumors, may be confined inside biological tissues (Stylianopoulos et al., 2012). In this context, cells develop compressive, growth-induced, mechanical stress (Fig. 1). Despite their paramount importance (Alessandri et al., 2013; Delarue et al., 2013, 2014; Fernández-Sánchez et al., 2015; Helmlinger, Netti, Lichtenbeld, Melder, \& Jain, 1997; Stylianopoulos et al., 2012; Tse et al., 2012), compressive mechanical stresses have been much less explored than other types of mechanical stress, for example tensile stresses (Butcher, Alliston, \& Weaver, 2009; Engler, Sen, Sweeney, \& Discher, 2006; Fletcher \& Mullins, 2010; Huang, Chen, \& Ingber, 1998; Levental et al., 2009; Northey, Przybyla, \& Weaver, 2017; Paszek et al., 2005), owing in part to the technical challenges of confining cells, and in particular microbes.

Several approaches were used in the past to study cells under confinement. In a pioneering study, Helmlinger and colleagues embedded mammalian cells in an agarose gel in order to study the impact of growth-induced pressure (Helmlinger et al., 1997). This approach had drawbacks, in particular the poor characterization of the mechanical properties of the agarose gel, making the evaluation of growth-induced pressure complicated to assess. More recently, Alessandri and colleagues overcame these drawbacks by embedding mammalian cells in a fully-mechanically characterized alginate shell (Alessandri et al., 2013). However, both approaches cannot readily be transferred to the study of microbes under confinement because the dimensions and elasticity of hydrogels is not compatible with the biophysical characteristics of microbes. Notably the amount of growth-induced stress microbes can build up can be orders of magnitude higher than that of mammalian cells, in the 100kPa range (Delarue et al., 2016) as opposed to the $1 \mathrm{kPa}$ range (Dolega et al., 2017; Farge, 2003) for mammalian cells. Moreover, the cell population was fully confined in both methodologies, a situation that may not always resemble the natural habitat of microbes.

We have employed the large repertoire of tools offered by microfluidics (Autebert et al., 2012; Glawdel, Elbuken, Lee, \& Ren, 2009; Groisman et al., 2005) to design several devices for the study of fungal cells under spatial confinement. Our device is in contrast with existing methodologies that either deformed single $S$. cerevisiae cells (Mishra et al., 2017), or partially confined S. pombe cells (Minc, Boudaoud, \& Chang, 2009). However, they enable to study cellular response at the single cell level in situ. The model organism used to design these devices is the budding yeast Saccharomyces cerevisiae, an organism of roughly $5 \mu \mathrm{m}$ diameter with a thick elastic cell wall and a large turgor pressure of several bars (Hohmann, 2002). Our devices enable either active or passive modulation of spatial confinement, and can precisely control compressive 
stress, while keeping the chemical environment fixed. We termed these devices mechano-chemostats. This chapter is organized as follows: section 2 first presents the design and application of mechano-chemostats; in section 3, we discuss the differences between passive and active devices, as well as how to measure growth-induced pressure; in section 4, some results obtained with these devices are presented; we finally discuss in section 5 potential future directions to create mechano-chemostats for other species.

\section{Presentation and microfabrication of the mechano-chemostat}

\subsection{Presentation of the device}

The mechano-chemostat consists of a confining chamber into which cells are loaded and can proliferate normally. The confining chamber is connected to a set of narrow channels too small for cells to enter (Fig. 2): These channels are used for media influx and efflux, with a media replacement time of tens of seconds allowing for constant control of the chemical environment while maintaining cell confinement. Several rounds of cell division quickly fill the whole chamber which, at a size of $65 \mu \mathrm{m} \times 25 \mu \mathrm{m}$ $\mathrm{x} 10 \mu \mathrm{m}$ (length $\mathrm{x}$ width $\mathrm{x}$ height), can accommodate about hundred cells. When the chamber is fully filled with cells, the excess cells flow out of the chamber through a microfluidic valve. We designed different sets of valves, passive or active, that are detailed in the third section. These microfluidic "faucets" tune cell confinement in the confining chamber: when open, there is no confinement thus no growth-induced pressure, whereas when fully closed, total confinement results in the build-up of strong growth-induced compressive stress. Pressure is either measured directly or inferred from the deformation of the confining chamber.

\subsection{Fabrication protocol}

The microfabrication protocol is classic (Campo \& Greiner, 2007; Jo, Van Lerberghe, Motsegood, \& Beebe, 2000) but is reminded below (Fig. 3). The material listed is specific to the fabrication of the devices presented in this chapter. We do not list the a particular brand for the mask aligner or for the spin coater.

\subsubsection{Material and Equipment}

- Negative photoresist, SU8 2000.5 and SU8 2010 (Microchem)

- SU8 developer (Microchem)

- Isopropanol

- Acetone

- Polydimethylsiloxane and curing agent (Sylgard 184)

- Number 1 thickness glass

- Puncher $0.75 \mathrm{~mm}$ (Harris)

- Reactive Ion Etcher (RIE, Diener)

- Oven set at $60^{\circ} \mathrm{C}$

\subsubsection{Fabrication process}

1. A first layer of SU8 2000.5 negative photoresist is spin-coated onto a silicon wafer to attain $0.5 \mu \mathrm{m}$ of thickness, then pre-baked. Note that the baking temperatures and times depend on the thickness and on the type of photoresist used.

2. This first layer is insulated by UV illumination in a mask aligner, followed by a post-exposure bake and development. Attaining the $1 \mu \mathrm{m}$ resolution of the nutrient 
channels is challenging and that the parameters usually used with the SU8 photoresists will have to be adjusted. In particular, we find that a smaller exposure and vacuum contact are often needed.

3. The first layer is developed using SU8 developer and then washed with isopropanol.

4. A second layer of SU8 2010 is spin-coated on the wafer to attain a $10 \mu \mathrm{m}$ height. The edges are removed manually with a clean room paper and some acetone, followed by pre-baking. Again, pre-baking depends on the photoresist used.

5. Insulation of the second layer, followed by post exposure bake.

6. The second layer is developed using and the top structures and alignment are checked under a microscope. If the structures are good, hard baking is follows. The wafer is silanized with trichlorosilane by vapor deposition to prevent attachment from PDMS.

7. 1:10 Sylgard 184 PDMS is finally molded on the silicon wafer, holes are created with the puncher, and the PDMS is bound to a \#1 thickness glass by oxygen plasma activation with a reactive ion etcher (RIE) reactor. Typical parameters used are: $100 \mathrm{~W}, \mathrm{P}_{02}=260 \mathrm{mbar}$, exposure time of 30s. When binding the PDMS to the glass, do not press it against it, as this may result in structures collapsing. Quickly place the device in an oven at $60^{\circ} \mathrm{C}$ for at least 20 minutes.

Note that the optimization of step 7 is crucial and may depend on the RIE used. Particularly important parameters to adjust are the partial pressure of oxygen and the reaction time. You can ensure that the binding is optimal by trying to rip it manually. When the binding is good, it is not possible to detach PDMS from the glass and you should end up breaking the PDMS and observing traces of it on the glass slide.

\subsection{Loading cells and imaging in the device}

We present in the following how to load the device and the type of imaging that can be performed.

\subsubsection{Material and equipment}

- $\quad$ PDMS device prepared in 2.2

- Cells grown overnight in exponential phase

- SCD medium

- Sterile $1 \mathrm{~mL}$ syringes (Terumo)

- Sterile 23G blunt needles (Strategic Applications Inc)

- PTFE microbore tubing 0.22x0.42in (Cole-Parmer)

\subsubsection{Loading of the device}

After connecting one of the main inlets to a syringe containing cells at an OD $\approx 0.4$, connect one of the two inlets for nutrients to a syringe containing the desired culture medium, and the other one to a tube to dispose of the waste (Fig. 4a). The loading of the device has to be performed in a specific sequence to avoid introduction of any air bubbles (Fig 4b):

1. Apply pressure on the chemical syringe to fill both the outlet and the cell inlet with medium.

2. While keeping some pressure on the chemical syringe to avoid air coming back into this channel, apply pressure on the cell syringe, to chase the air through the outlet. At some point, air will be flowing out of the device through the outlet.

3. Only apply pressure on the cell syringe, to load the device with the desired amount of cells. Most of our experiments started with 1-5 cells. Loading the device can be 
stressful for the cells, so starting the experiment with just a few cells and allowing them to recover while they are filling the chamber ensures better reproducibility.

4. When the device is loaded, take out the cell syringe and its tubing, and seal this inlet with a plug, for instance made out of a melted tubing. Apply a steady flow that can be imposed with your favorite syringe pump, through the chemical inlet: because the cell inlet is closed, the medium will mainly flow through the chamber and the outlet.

\subsubsection{Imaging}

All of our devices are bound to \#1 thickness glass. PDMS being transparent, this allows for imaging, both in bright field and in fluorescence. We have used magnification from 40x to 100x, and imaging methods such as bright field, phase contrast, epifluorescence, confocal microscopy, fluorescence correlation spectroscopy (FCS), fluorescence recovery after photobleaching (FRAP), and even performed laser-ablation in situ. Because we can control the chemical environment, most of the classical biological staining techniques, such as immunofluorescence staining or FISH are also compatible with our devices and allow for precise analysis of the response to compressive stress. Hence, there is no particular method associated with imaging.

\section{Control of the mechanical environment}

We designed valves of different kinds to limit the cell outflow from the confining chamber to impose a given degree of confinement to the cell population. Passive valves limit the flow without the use for external drive, in contrast with active valves which are water-driven pneumatics.

\subsection{Passive valves}

\subsubsection{Material and equipment}

No particular equipment is needed to use the passive valves.

\subsubsection{Using the passive valves}

We designed a set of various obstacles that naturally limit cell outflow (Fig. 5a). We found that obstacles work by inducing jamming in the cell population (Delarue et al., 2016): force chains spanning the whole population stabilize it, leading to partial confinement and a build-up of growth-induced pressure. Pressure rises until the force network is broken, leading to an avalanche of cells through the outlet and a decrease of pressure. Over longer time, the geometry of the obstacle sets the mean steady-state pressure around which the cell population will stochastically vary. We also took advantage of the propensity of $S$. cerevisiae cells to jam to design a valve that fully confines the cell population (Fig. 5b). When cells fill the chamber, growth-induced pressure builds up through jamming and results in the deformation of PDMS. By adding side-pockets that surround the outlet channel to the confining chamber, PDMS deformation will result in these side-pockets pinching the exit channel, completely confining cells. This total confinement results in stronger jamming, and a larger pressure build-up, up to a stall pressure for cell growth.

Hence, the use of these valves to impose a growth-induced compressive stress does not require any external drive, and can be used with the devices prepared in Section 2 without further equipment. The deformation of the device informs on the amount of compressive stress - see 3.3 below. 


\subsection{Active valves}

\subsubsection{Material and equipment}

- Loaded device (see Section 2)

- Syringe pump (Cetoni $\mathrm{GmbH})$

- Pressure sensor (Cetoni $\mathrm{GmbH})$

\subsubsection{Using the active valves}

The approach with the side pockets surrounding the outlet can be extended to design an externally water-driven valve. We surrounded the outlet with two vertical membranes that can be actuated with a measured water-driven pressure (Fig. 5c). The range of pressure being up to several bars, we chose to use a syringe pump coupled to a pressure sensor to impose a given pressure knowing the volume flown in the chamber, instead of a pressure pump that is limited in the maximum amount of pressure that can be applied when compressing air.

The deformation of the membranes by water-driven pressure can be used to pinch the outlet channel. When the outlet channel is open, the cell population is not confined and no pressure builds up. Conversely, when the channel is closed, cell outflow becomes limited and growth accumulates in the chamber, resulting in a progressive growth-induced pressure. We found a linear correlation between the closing of the valve and the intensity of growth-induced pressure (Delarue et al., 2017). This valve thus functions as a microfluidic "faucet". This type of valve is useful to precisely and dynamically control the intensity of growth-induced pressure in the chamber.

\subsection{Measuring pressure}

\subsubsection{Material and equipment}

- Loaded device (see Section 2)

- Syringe pump (Cetoni $\mathrm{GmbH})$

- Pressure sensor (Cetoni $\mathrm{GmbH})$

\subsubsection{Pressure measurement}

The confining chamber is in contact with a vertical membrane, a "pressure transducer" (Fig. 2) deformed by the build-up of growth-induced pressure. Growth-induced pressure can be measured in two ways:

1. Careful calibration of the deformation of the membrane by a given water-driven pressure before the experiment enables calculation of the pressure developed by the cell population by measuring the deformation of the membrane. We find a linear relationship between the deformation $\delta$ and the pressure $\mathrm{P}$ for a given Young modulus E: $\delta=12.09 \mathrm{P} / \mathrm{E}$ in $\mu \mathrm{m}$ (Delarue et al., 2016).

2. The water-driven pressure applied on the membrane can be adjusted to keep the membrane at the same position at all time: when at mechanical equilibrium, the water-driven pressure matches the growth-induced pressure, providing a direct readout of the mechanical stress developed by the cell population.

Both modes present advantages and drawbacks. The first mode requires measurement of the mechanical properties of the PDMS beforehand but allows for rapid measurement of pressure changes that may occur through sudden avalanches of the cell population. In contrast, the second mode does not require any calibration, but requires a complex feedback loop where software has to control both the camera for image acquisition and 
the pressure pumps. We used Matlab to perform these operations, which is compatible with most commercial cameras and pressure pump drivers.

\subsection{Micropiston and instantaneous compressive stress}

\subsubsection{Material and Equipment}

- Loaded device (see Section 2)

- $\quad$ Syringe pump (Cetoni $\mathrm{GmbH})$

- Pressure sensor (Cetoni $\mathrm{GmbH})$

\subsubsection{Applying an instantaneous pressure}

We used the pressure transducer to directly and instantaneously compress the cell population (Delarue et al., 2017): By increasing the water-driven pressure, one can deform the membrane in order to compress the cell population (Fig. $5 \mathrm{c}$ ). Note that here the pressure that the cells experience does not directly correspond the pressure applied to the pressure transducer, as the pressure experienced would depend on the elastic properties of the PDMS and the packing fraction of cells. Hence pressure sensors such as the ones described in (Dolega et al., 2017) should be used in combination to this method in order to accurately measure the intensity of this instantaneous pressure.

\subsection{Passive or active valves?}

Each type of device has pros and cons. The passive valve devices are fast and easy-touse and enable a large range of growth-induced pressure through the use of outlets of various geometries. We have successfully parallelized passive valve devices to enable high-throughput analysis (Delarue et al., 2017). Their disadvantage over the active valves is the lack of dynamic control of the pressure: once the cells in a device are enclosed by a passive valve, pressure can only build up to an extent imposed by the geometry of the valve. The use of active valves requires more specialized equipment like pressure or syringe pumps and hardware control. However, their versatility is excellent: growth-induced pressure can be dynamically controlled, where different pressure intensities can be tested during the same experiment on the same cell population to test for mechanical adaptation, and pressure can even be relaxed in order to study the reversibility of observed phenotypes.

\section{Physiological response to a mechanical stress}

In this section we present two different aspects of the impact of mechanical stress on cell physiology that we obtained using the previously described mechano-chemostats.

\subsection{Growth-induced pressure limits both cell growth and cell proliferation}

Growth rate in the mechano-chemostat can be measured at a steady-state pressure by the flow rate of cells leaving the chamber. We measured that this rate decreases with increasing compressive stress (Fig. 6a). In parallel, we found that cells under pressure are delayed at the beginning of the cell cycle, in a cell cycle phase termed G1 (Fig. 6b). It is worthwhile noting that both behaviors have also been observed in compressed mammalian cells (Montel et al., 2011, 2012). Whereas the decrease in growth rate comes from either active biological regulation or passive physical response to compressive stress, the decrease in cell proliferation is a biological response, suggesting 
that these unicellular organisms are capable of sensing and responding to mechanical stress.

\subsection{The SCWISh network is essential for survival under growth- induced pressure}

We used a high-throughput version of the passive devices in order to rapidly screen for mutants essential for cell survival under compressive stress. We found that the transmembrane mucin Msb2p, previously described as an osmosensor (Saito \& Posas, 2012), was also a mechano-sensor of growth-induced compressive stress. Together with the scaffold protein Sholp this mucin triggers the activation of the MAPKKK Ste11p to drive partial adaptation to compressive stress. We termed this pathway $S M u S h$, for Ste11p activation through Mucin Msb2p and Sho1p (Fig. 7a). Both (Delarue et al., 2017; Mishra et al., 2017) elucidated a role for the cell wall integrity pathway in the response to compressive stress. The cell wall integrity pathway $(C W I)$ and $S M u S h$ pathway act in parallel: abrogation of both $C W I$ and SMuSh pathways totally abolishes cell survival under compressive stress (Fig. 7b). We named this network SCWISh (Survival through $C W I$ and SMuSh). The SCWISh network is essential for cells to fully adapt and survive under mechanical pressure.

\section{Discussion}

The proposed mechano-chemostats are extremely flexible microfluidic devices that enable the study of compressive mechanical stress. They are compatible with optical imaging, and the control of the chemical environment makes it possible to do staining like FISH or immunofluorescence in situ. Moreover, high-throughput devices can be used to rapidly screen for multiple conditions, either genetic or chemical, that would alter cell proliferation/survival under a compressive mechanical stress.

Even though they were developed for the fungus $S$. cerevisiae, we were also able to use them to study other yeasts including $S$. pombe and $C$. albicans. The geometry of mechano-chemostats, notably the valve and the size of the nutrient channels, can either be downscaled to study smaller microbial organisms, or upscaled for the study of mammalian cells. The advantage of mechano-chemostats over existing techniques (Alessandri et al., 2013; Helmlinger et al., 1997; Minc et al., 2009; Mishra et al., 2017) resides in the versatility of their usage. They allow dynamic control of pressure, along with real-time control of the chemical environment. We believe that our approach could be adapted to ask a variety of fundamental questions regarding the impact of compressive stress on living organisms.

\section{References}

Alessandri, K., Sarangi, B. R., Gurchenkov, V. V., Sinha, B., Kießling, T. R., Fetler, L., ... Nassoy, P. (2013). Cellular capsules as a tool for multicellular spheroid production and for investigating the mechanics of tumor progression in vitro. Proceedings of the National Academy of Sciences of the United States of America, 110(37), 14843-14848. https://doi.org/10.1073/pnas.1309482110

Autebert, J., Coudert, B., Bidard, F.-C., Pierga, J.-Y., Descroix, S., Malaquin, L., \& Viovy, J.-L. (2012). Microfluidic: an innovative tool for efficient cell sorting. Methods, 57(3), 297-307.

Butcher, D. T., Alliston, T., \& Weaver, V. M. (2009). A tense situation: forcing tumour progression. Nature Reviews Cancer, 9(2), 108-122. 
Campo, A. del, \& Greiner, C. (2007). SU-8: a photoresist for high-aspect-ratio and 3D submicron lithography. Journal of Micromechanics and Microengineering, 17(6), R81-R95. https://doi.org/10.1088/0960-1317/17/6/R01

Delarue, M., Hartung, J., Schreck, C., Gniewek, P., Hu, L., Herminghaus, S., \& Hallatschek, O. (2016). Self-driven jamming in growing microbial populations. Nature Physics, 12(8), 762-766. https://doi.org/10.1038/nphys3741

Delarue, M., Montel, F., Caen, O., Elgeti, J., Siaugue, J.-M., Vignjevic, D., ... Cappello, G. (2013). Mechanical Control of Cell flow in Multicellular Spheroids. Physical Review Letters, 110(13), 138103. https://doi.org/10.1103/PhysRevLett.110.138103

Delarue, M., Montel, F., Vignjevic, D., Prost, J., Joanny, J.-F., \& Cappello, G. (2014). Compressive Stress Inhibits Proliferation in Tumor Spheroids through a Volume Limitation. Biophysical Journal, 107(8), 1821-1828. https://doi.org/10.1016/j.bpj.2014.08.031

Delarue, M., Poterewicz, G., Hoxha, O., Choi, J., Yoo, W., Kayser, J., ... Hallatschek, O. (2017). SCWISh network is essential for survival under mechanical pressure. Proceedings of the National Academy of Sciences of the United States of America, 114(51), 13465-13470. https://doi.org/10.1073/pnas.1711204114

Dolega, M. E., Delarue, M., Ingremeau, F., Prost, J., Delon, A., \& Cappello, G. (2017). Cell-like pressure sensors reveal increase of mechanical stress towards the core of multicellular spheroids under compression. Nature Communications, 8, 14056. https://doi.org/10.1038/ncomms 14056

Engler, A. J., Sen, S., Sweeney, H. L., \& Discher, D. E. (2006). Matrix elasticity directs stem cell lineage specification. Cell, 126(4), 677-689.

Farge, E. (2003). Mechanical induction of Twist in the Drosophila foregut/stomodeal primordium. Current Biology: $C B, 13(16), 1365-1377$.

Fernández-Sánchez, M. E., Barbier, S., Whitehead, J., Béalle, G., Michel, A., LatorreOssa, H., ... Farge, E. (2015). Mechanical induction of the tumorigenic $\beta$-catenin pathway by tumour growth pressure. Nature, 523(7558), 92-95.

Fletcher, D. A., \& Mullins, R. D. (2010). Cell mechanics and the cytoskeleton. Nature, 463(7280), 485-492. https://doi.org/10.1038/nature08908

Glawdel, T., Elbuken, C., Lee, L. E. J., \& Ren, C. L. (2009). Microfluidic system with integrated electroosmotic pumps, concentration gradient generator and fish cell line (RTgill-W1)--towards water toxicity testing. Lab on a Chip, 9(22), 32433250. https://doi.org/10.1039/b911412m

Groisman, A., Lobo, C., Cho, H., Campbell, J. K., Dufour, Y. S., Stevens, A. M., \& Levchenko, A. (2005). A microfluidic chemostat for experiments with bacterial and yeast cells. Nature Methods, 2(9), 685-689. https://doi.org/10.1038/nmeth784

Helmlinger, G., Netti, P. A., Lichtenbeld, H. C., Melder, R. J., \& Jain, R. K. (1997). Solid Stress inhibits the growth of multicelular tumor spheroids. Nature Biotechnology, 15, 778-783.

Hohmann, S. (2002). Osmotic Stress Signaling and Osmoadaptation in Yeasts Osmotic Stress Signaling and Osmoadaptation in Yeasts. Microbiol Mol Biol $R E v, 66(2)$. https://doi.org/10.1128/MMBR.66.2.300

Huang, S., Chen, C. S., \& Ingber, D. E. (1998). Control of Cyclin D1, p27Kip1, and Cell Cycle Progression in Human Capillary Endothelial Cells by Cell Shape and Cytoskeletal Tension. Molecular Biology of the Cell, 9(11), 3179-3193. https://doi.org/10.1091/mbc.9.11.3179

Jo, B.-H., Van Lerberghe, L. M., Motsegood, K. M., \& Beebe, D. J. (2000). Three- 
dimensional micro-channel fabrication in polydimethylsiloxane (PDMS) elastomer. Journal of Microelectromechanical Systems, 9(1), 76-81. https://doi.org/10.1109/84.825780

Levental, K. R., Yu, H., Kass, L., Lakins, J. N., Egeblad, M., Erler, J. T., ... Weaver, V. M. (2009). Matrix Crosslinking Forces Tumor Progression by Enhancing Integrin Signaling. Cell, 139(5), 891-906. https://doi.org/10.1016/j.cell.2009.10.027

Minc, N., Boudaoud, A., \& Chang, F. (2009). Mechanical forces of fission yeast growth. Current Biology : CB, 19(13), 1096-1101. https://doi.org/10.1016/j.cub.2009.05.031

Mishra, R., van Drogen, F., Dechant, R., Oh, S., Jeon, N. L., Lee, S. S., \& Peter, M. (2017). Protein kinase $C$ and calcineurin cooperatively mediate cell survival under compressive mechanical stress. Proceedings of the National Academy of Sciences of the United States of America, 114(51), 13471-13476. https://doi.org/10.1073/pnas.1709079114

Montel, F., Delarue, M., Elgeti, J., Malaquin, L., Basan, M., Risler, T., ... Joanny, J.F. (2011). Stress Clamp Experiments on Multicellular Tumor Spheroids. Physical Review Letters, 107(18), 188102. https://doi.org/10.1103/PhysRevLett.107.188102

Montel, F., Delarue, M., Elgeti, J., Vignjevic, D., Cappello, G., \& Prost, J. (2012). Isotropic stress reduces cell proliferation in tumor spheroids. New Journal of Physics, 14(5), 55008. https://doi.org/10.1088/1367-2630/14/5/055008

Northey, J. J., Przybyla, L., \& Weaver, V. M. (2017). Tissue force programs cell fate and tumor aggression. Cancer Discovery, 7(11), 1224-1237. https://doi.org/10.1158/2159-8290.CD-16-0733

Paszek, M. J., Zahir, N., Johnson, K. R., Lakins, J. N., Rozenberg, G. I., Gefen, A., ... Weaver, V. M. (2005). Tensional homeostasis and the malignant phenotype. Cancer Cell, 8(3), 241-254. https://doi.org/10.1016/j.ccr.2005.08.010

Saito, H., \& Posas, F. (2012). Response to hyperosmotic stress. Genetics, 192(2), 289-318. https://doi.org/10.1534/genetics.112.140863

Stylianopoulos, T., Martin, J. D., Chauhan, V. P., Jain, S. R., Diop-Frimpong, B., Bardeesy, N., ... Jain, R. K. (2012). Causes, consequences, and remedies for growth-induced solid stress in murine and human tumors. Proceedings of the National Academy of Sciences of the United States of America, 109(38), 1510115108. https://doi.org/10.1073/pnas.1213353109

Tse, J. M., Cheng, G., Tyrrell, J. A., Wilcox-Adelman, S. A., Boucher, Y., Jain, R. K., \& Munn, L. L. (2012). Mechanical compression drives cancer cells toward invasive phenotype. Proceedings of the National Academy of Sciences of the United States of America, 109(3), 911-916. https://doi.org/10.1073/pnas.1118910109

Warscheid, T., \& Braams, J. (2000). Biodeterioration of stone: a review. International Biodeterioration \& Biodegradation, 46(4), 343-368. https://doi.org/10.1016/S0964-8305(00)00109-8 


\section{Figures}

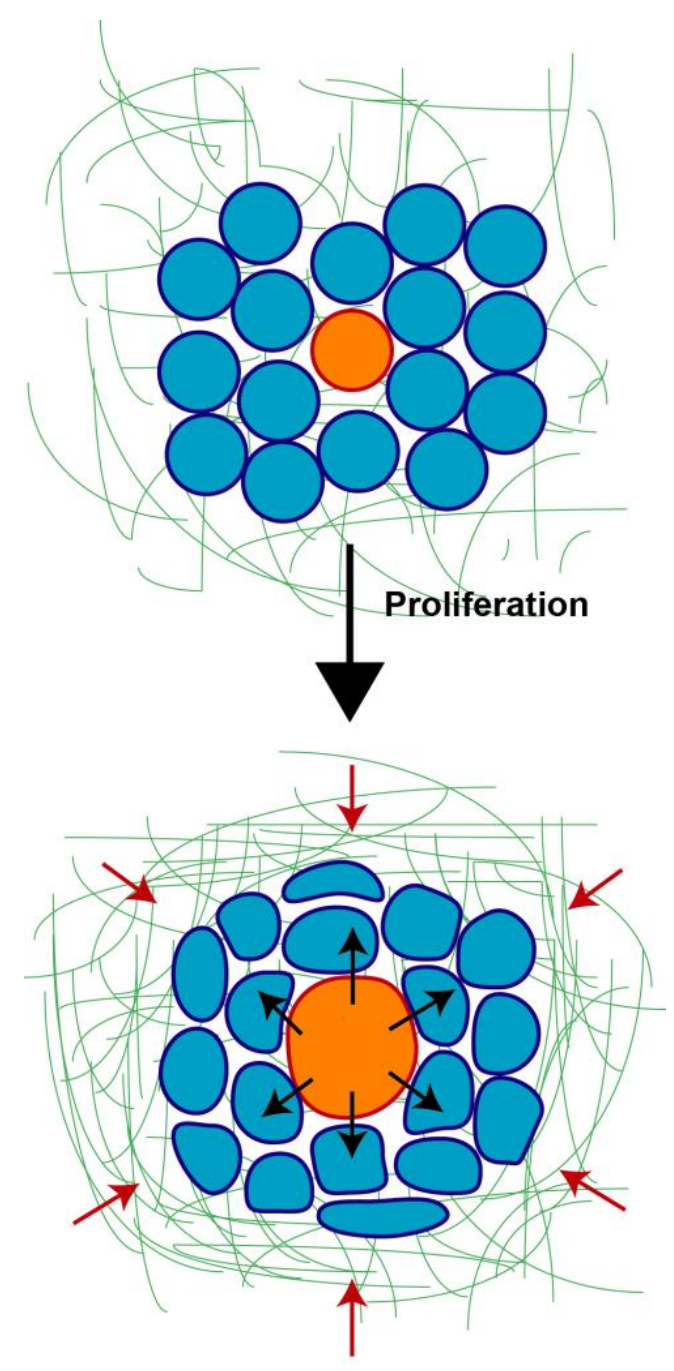

\& elastic extracellular matrix

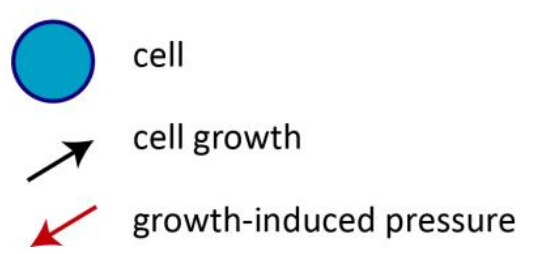

Figure 1

When growing in a spatially-constrained environment, cells need to act upon their surroundings, for example other cells and an elastic matrix, to accommodate space for new cell material. Growth-induced contact pressures emerge naturally under these conditions. 


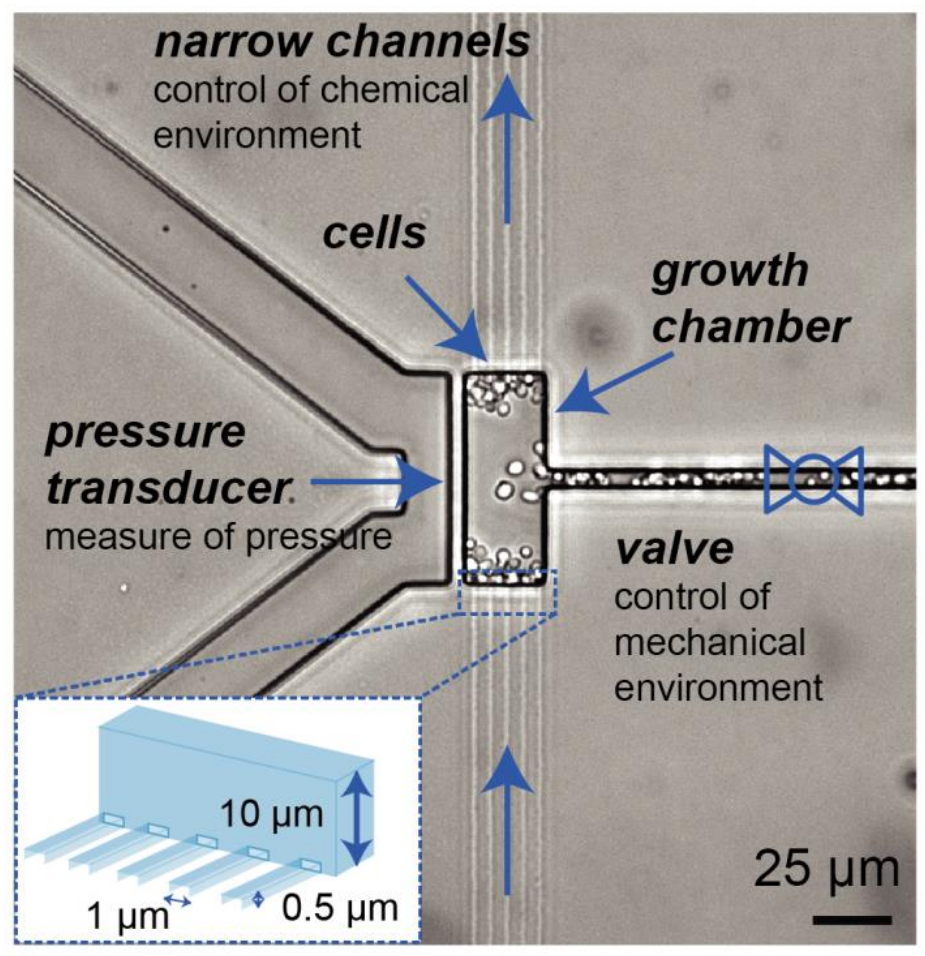

\section{Figure 2}

Design of a mechano-chemostat. Cells are cultured in a confining chamber that is connected to a set of narrow channels that are too small for cells to enter. These channels are used to set the chemical environment. Cell outflow is limited by a valve that partially or totally confines the population, thus modulating growth-induced compression, which is measured by the pressure transducer. The valves can be active or passive, as illustrated in Fig. 5. 




\section{Figure 3}

Microfabrication steps. Classical photolithography is used to create silicon molds in two steps: the thin layer that defines geometry including nutrient channels first, then the second thicker layer that completes the growth chambers and input channels. 


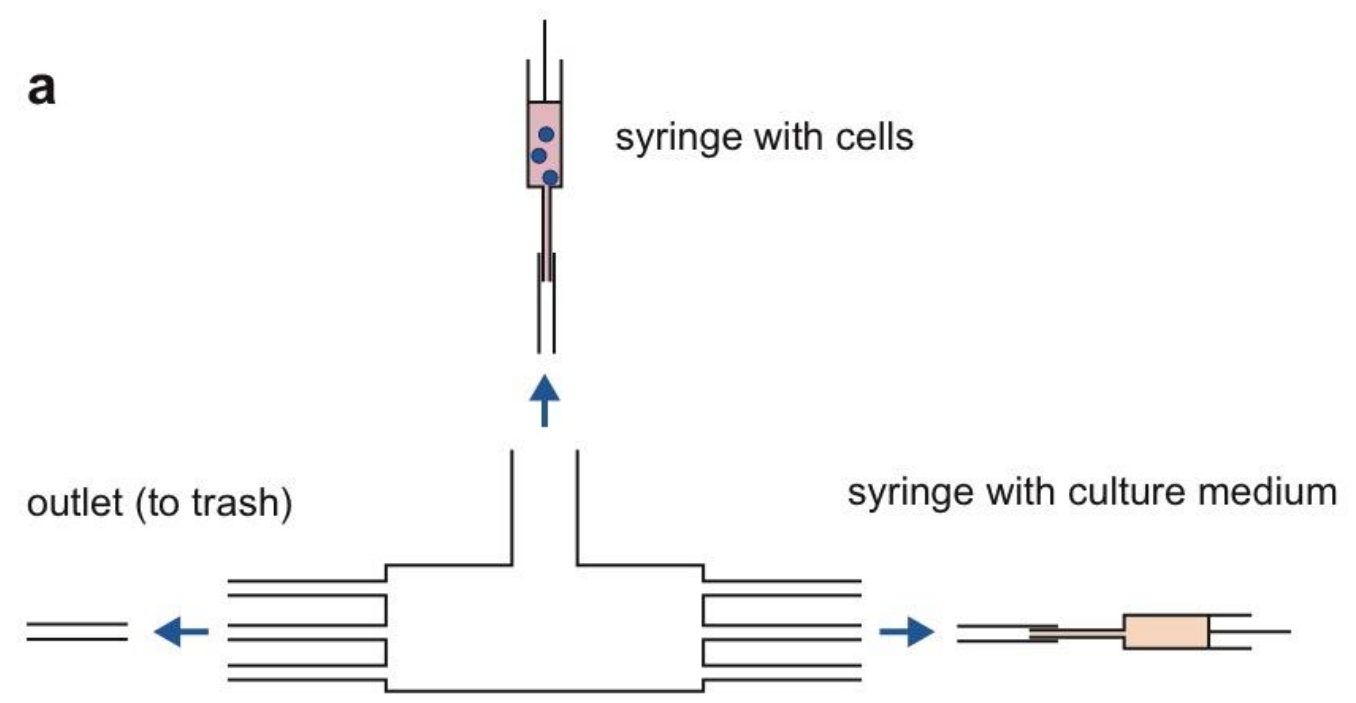

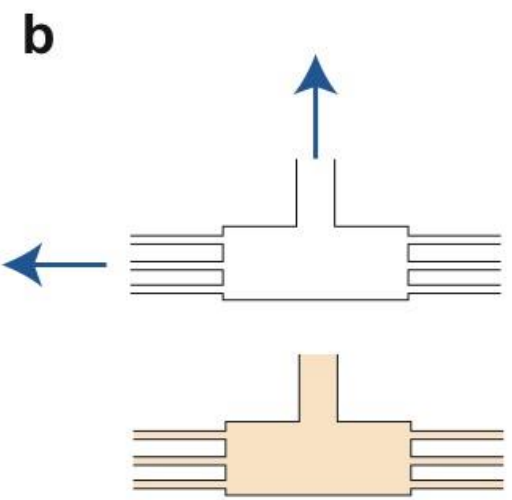

1. Injection of culture medium

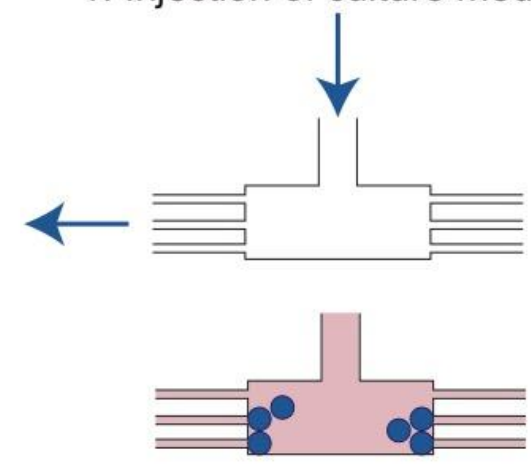

3. Injection of cells
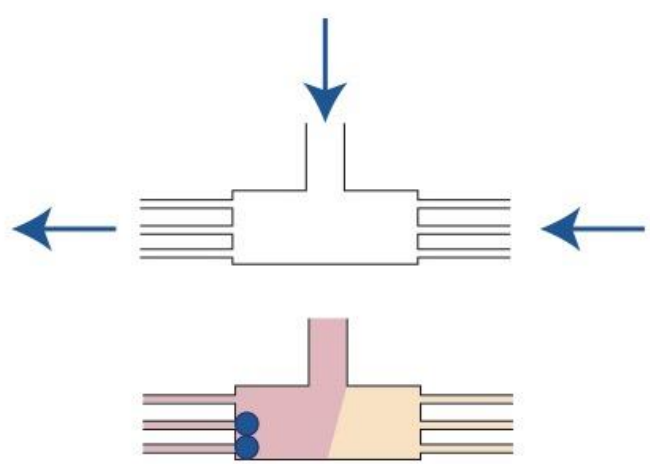

2. Injection of cells and culture medium

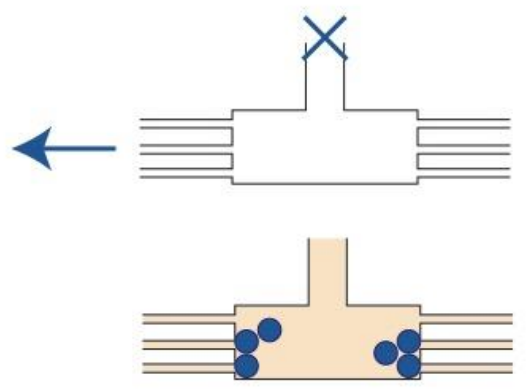

4. Steady-flow of culture medium

\section{Figure 4}

Loading of the device has to occur in a correct order. a. Plug the syringes in the correct holes. b. Load the medium first, then the cells, to fully get rid of air. 


\section{Passive valves}

a

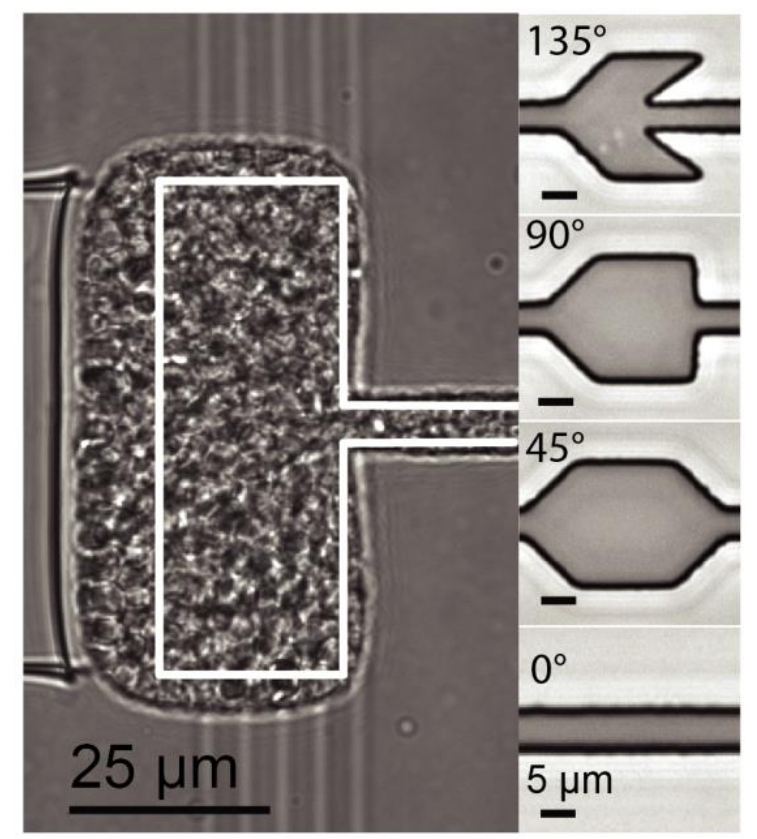

Active valves

C
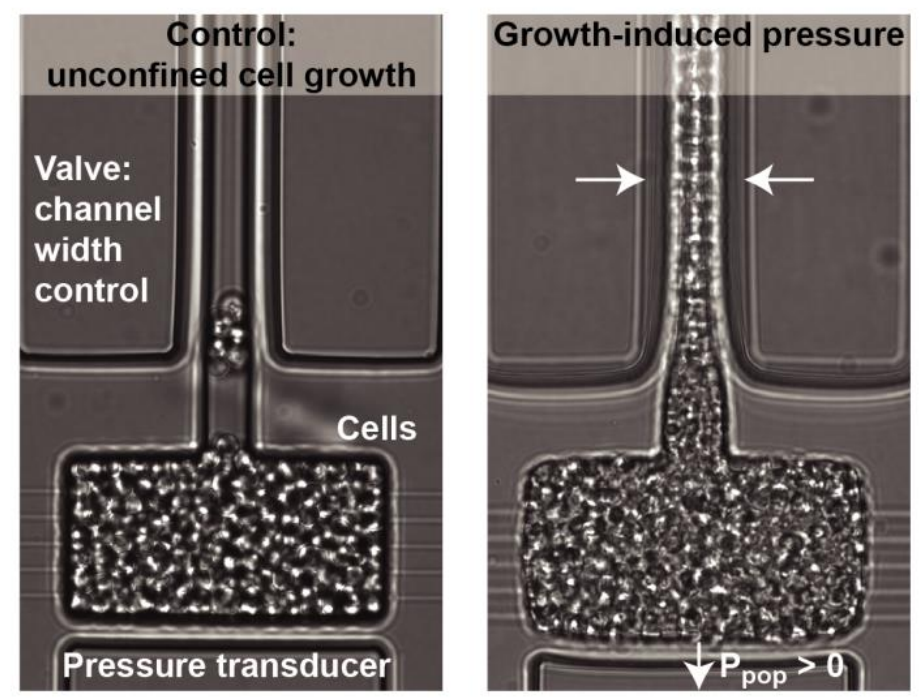
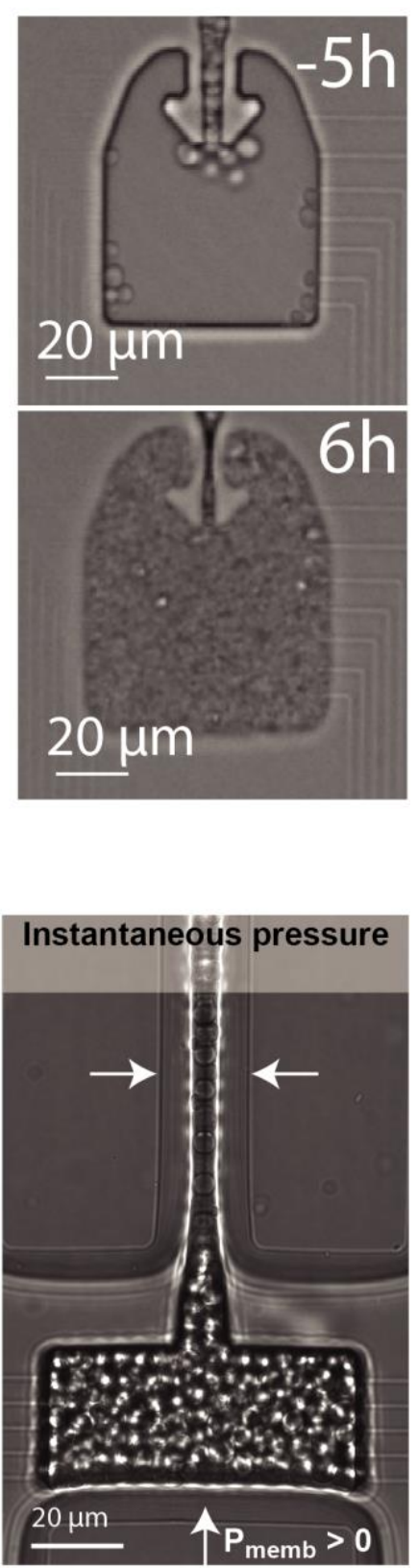

\section{Figure 5}

Different types of valves are connected to the confining chamber. a. Passive valves with various geometries will limit the flow and increase pressure through self-driven jamming. b. A self-closing valve can be used to fully confine the cell population. c. Vertical membranes can be actuated by a water-driven pressure to close the outlet channel to a desired amount, resulting in the build-up of growth-induced pressure. The pressure transducer can be used to either measure pressure through its deformation or used as a micropiston to further compress the cell population. 
a

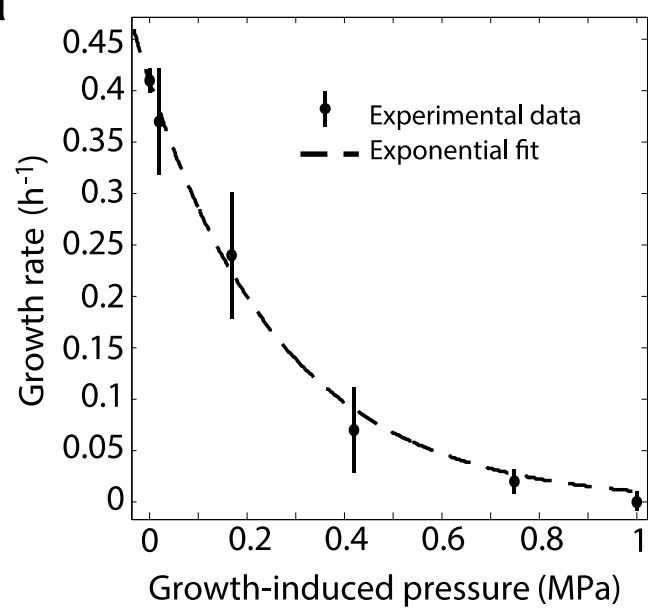

b

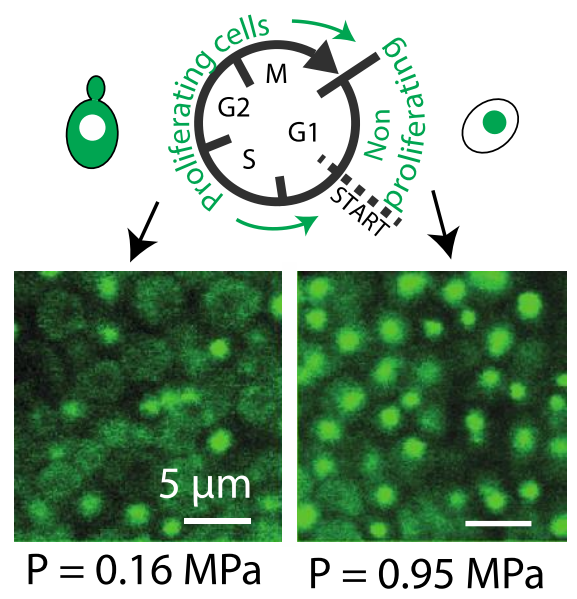

\section{Figure 6}

Impact of compressive stresses on cell proliferation. a. Growth rate decreases roughly exponentially as a function of growth-induced pressure. b. Growth-induced pressure blocks the cells in the G1 phase of the cell cycle. 
a

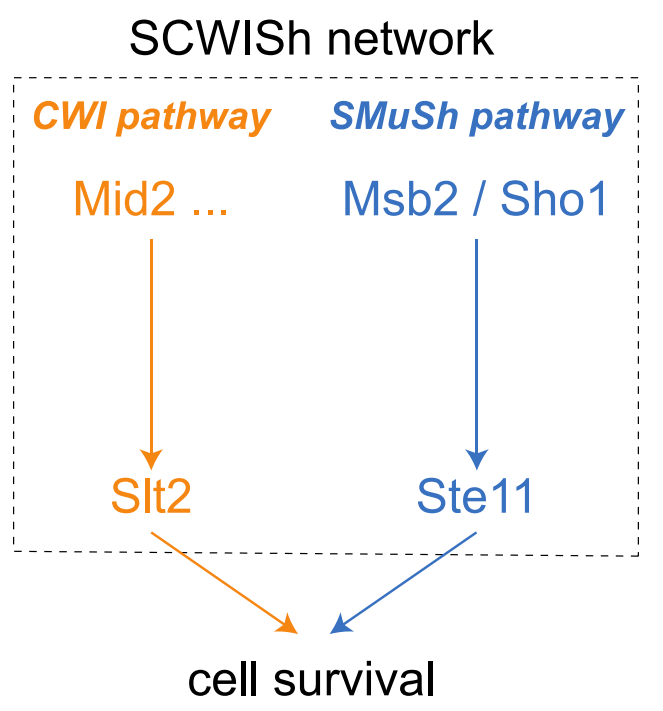

b

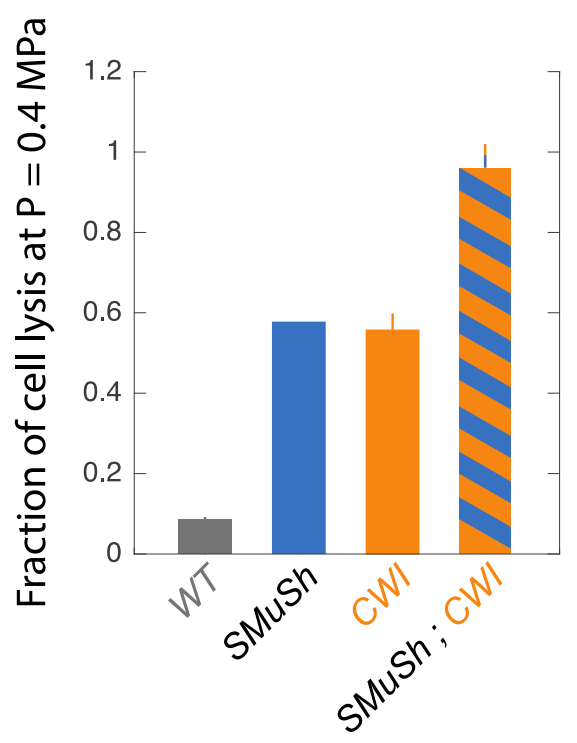

\section{Figure 7}

The SCWISh network is essential for cell survival under pressure. a. The mechanosensitive $C W I$ and SMuSh pathways define the SCWISh network. b. Abrogation of both $C W I$ and $S M u S h$ pathways results in total cell death under compressive stress. 